\title{
Next-Generation Sequencing and Epigenomics Research: A Hammer in Search of Nails
}

\author{
Shrutii Sarda*, Sridhar Hannenhalli** \\ Center for Bioinformatics and Computational Biology, University of Maryland, College Park, MD 20740, USA
}

\begin{abstract}
After the initial enthusiasm of the human genome project, it became clear that without additional data pertaining to the epigenome, i.e., how the genome is marked at specific developmental periods, in different tissues, as well as across individuals and species - the promise of the genome sequencing project in understanding biology cannot be fulfilled. This realization prompted several large-scale efforts to map the epigenome, most notably the Encyclopedia of DNA Elements (ENCODE) project. While there is essentially a single genome in an individual, there are hundreds of epigenomes, corresponding to various types of epigenomic marks at different developmental times and in multiple tissue types. Unprecedented advances in next-generation sequencing (NGS) technologies, by virtue of low cost and high speeds that continue to improve at a rate beyond what is anticipated by Moore's law for computer hardware technologies, have revolutionized molecular biology and genetics research, and have in turn prompted innovative ways to reduce the problem of measuring cellular events involving DNA or RNA into a sequencing problem. In this article, we provide a brief overview of the epigenome, the various types of epigenomic data afforded by NGS, and some of the novel discoveries yielded by the epigenomics projects. We also provide ample references for the reader to get in-depth information on these topics.
\end{abstract}

Keywords: chromatin accessibility, epigenomics, methylation, next-generation sequencing, regulation

\section{Introduction}

Next-generation sequencing (NGS) has resulted in an exponential growth in data in the past decade, primarily fueled by the general interest of the scientific community in functionally annotating the human genome. The power of high-throughput parallel sequencing was deployed to characterize human genetic variation, in an international effort launched in 2008, called the 1000 Genomes Project, with the hope that such a large-scale endeavor would help in identifying all the underlying genetic differences that lead to disease resistance/susceptibility [1].

In the meantime, based on numerous genome-wide association studies (GWAS), it was realized that $93 \%$ of the trait-associated polymorphisms within the human population were located in non-coding regions, potentially within cis-regulatory elements [2]. In fact, in the mid-1970s, King and Wilson [3] pointed out the importance of mapping regulatory events and hinted that a small difference in the time/level of activation of a single gene could influence overall systemic development. They estimated the genetic distance between human and chimpanzee to be much smaller than the corresponding genetic distance between the sibling species of Drosophila, thereby underscoring the need to explore regulatory/expression differences at different times as a basis to explain the evolutionary differences at the organismal level [3].

\section{Why the Epigenome?}

A mechanistic understanding of spatio-temporal gene expression in eukaryotes is far from complete. While genetic differences might be expected to explain expression divergence across species and expression variability across individuals of a population, it cannot explain how almostidentical genomes in more than 200 different cell types within an individual organism can drive such varied expression profiles specific to each cell type [4]. DNA is packaged 


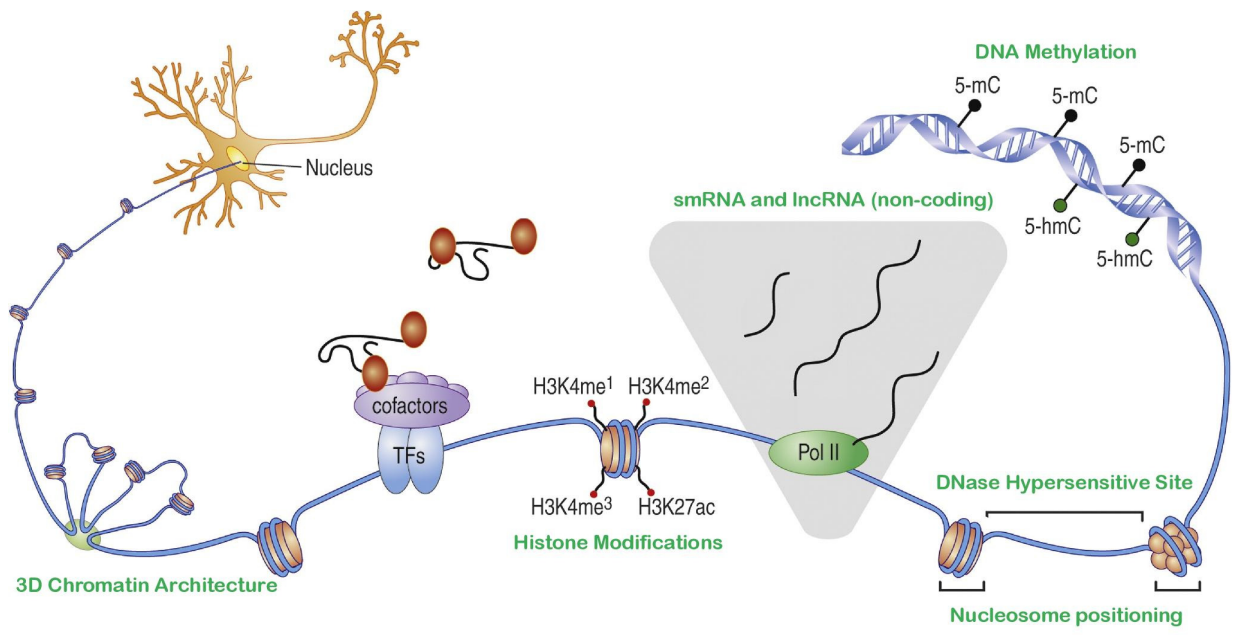

Fig. 1. A schematic depicting the context of several epigenetic marks on chromatin; ranging from DNA methylation, nucleosome positioning patterns affecting the size and distribution of DNase I hypersensitive sites, transcriptional activity at non-coding sites leading to the production of small and long RNA, chemical modifications of histone moieties (e.g., mono-, di- and tri-methylation of the 4th lysine of the $\mathrm{H} 3$ subunit), and chromatin folding to form localized structures in 3D nuclear space (anticlockwise). Adapted from Fig. 1 in Telese et al. [9], Copyright(C)2013, with permission from Elsevier. in a nucleoprotein complex structure called chromatin that is highly dynamic, in that its "states" vary from one cell type to another. Therefore, a possible solution to this conundrum is to analyze these "states" at the genome level, i.e., characterize the landscape of the epigenome, which further qualifies and modulates the functional effect of genetic information [5]. Epigenome, meaning "on or above genomes," refers to such sequence-independent heritable properties of the genome that can modulate the functional output of the genome. While, as described initially, heritability is an important qualifying criterion of an epigenomic mark (most notably, DNA methylation) [6], currently, the term epigenome is loosely used to encompass a myriad of chemical changes to DNA or histone proteins, chromatin accessibility, small and long (non-coding) RNA localization, and higher-order DNA organization (including nucleosome occupancy and positioning, and 3D chromatin interactions) (Fig. 1) [7-9]. The ultimate combined effect of the epigenome is to determine the transcriptome (the set of all transcripts) of a cell precisely. Epigenomic features influence the regulatory program of each gene's expression in several ways: they define the local environment of specific processes by regulating the chromatin architecture, determine access of transcription factors to DNA, as well as serve to keep a "memory" of cell type-specific features facilitating heritability of epigenetic characteristics $[10,11]$.

\section{The Wild West of Epigenetic Marks and the Impact of NGS Techonologies}

The will of the global scientific community to sequence the human genome spurred advances in sequencing technologies that led to what is now known as NGS. The improvement in cost and speed of sequencing in the last decade has significantly surpassed the analogous improvement in computer technologies as predicted by Moore's law [12]. Well beyond the initial goal of sequencing complete genomes of several species, NGS is now routinely used as a tool to investigate the diverse array of cellular events involving DNA or RNA, such as the identification of genomic loci bound by a specific protein, the detection of pairs of spatially proximal or interacting chromosomal loci, etc. Broad availability of NGS technologies has led to a paradigm shift in molecular biology research; from probing singular cellular events to an unbiased genome-scale mapping of such events, and has enabled genome-wide elucidation of the epigenomic landscapes in hundreds of cell types, across developmental times, in human, as well as other species [13].

As mentioned earlier, it is the locus-specific "epigenetic code" (a specific signature or combination of several epigenetic modifications) that helps define a cell's expression program and identity [14], thereby distinguishing it from other cell types. Unlike the largely static genome of an individual, the epigenome is highly variable and dynamic; yet, many of these marks can be passed down a cell's lineage, or from one generation to the next. In this section, we describe several well-studied epigenomic marks and outline what is known about their role in gene expression modulation. We will also outline various NGS-based assays that are used in comprehensively mapping each of the epigenetic marks (Table 1) [15-34].

\section{DNA methylation}

One of the more stable and heritable epigenetic marks is DNA methylation. The human genome is highly methylated; approximately $80 \%$ of cytosines in CpG dinucleotides are chemically modified at their fifth carbon atom with a methyl group [35]. Historically, DNA methylation was associated with transcriptional silencing (as evidenced by many promoter-based studies) [36]. Although genome-scale profiling 
Table 1. A summary of epigenetic marks, their types (wherever applicable), and the NGS-based assays used to map their location and distribution

\begin{tabular}{|c|c|c|}
\hline Broad epigenetic features & Types of marks (if applicable) & Assays \\
\hline DNA methylation & $\begin{array}{l}\text { 5-mC: methyl cytosine } \\
\text { Variants } \\
\text { 5-hmC: hydroxyl methyl cytosine } \\
\text { 5-fC: formyl cytosine } \\
\text { 5-caC: carboxyl cytosine }\end{array}$ & $\begin{array}{l}\text { Restriction based: MRE-seq [15] } \\
\text { Affinity based: MeDIP-seq [16] and MBD-seq [17] } \\
\text { Chemical based: RRBS [18] and WGBS/methylC-seq } \\
\text { [19] } \\
\text { oxBS-seq (to distinguish between 5-mC and 5-hmC) } \\
\text { [20] }\end{array}$ \\
\hline Histone modifications & $\begin{array}{l}\text { H3K27me3: associated with repressed regions } \\
\text { H3K4me1: associated with enhancers } \\
\text { H3K4me3: associated with promoters } \\
\text { H3K27ac: associated with active enhancers } \\
\text { H3K9ac: associated with active promoters } \\
\text { H3K36me3: associated with gene bodies } \\
\text { H3K9me3: associated with heterochromatin }\end{array}$ & $\begin{array}{l}\text { ChIP-seq }[21] \\
\text { ChIP-exo }[22]\end{array}$ \\
\hline $\begin{array}{l}\text { Nucleosome positioning and } \\
\text { occupancy }\end{array}$ & - & $\begin{array}{l}\text { MNase-seq [23] } \\
\text { MNase-independent mutated hisotones based } \\
\quad \text { mapping [24] }\end{array}$ \\
\hline Chromatin accessibility & - & $\begin{array}{l}\text { DNase-seq [25] } \\
\text { DGF [26] } \\
\text { FAIRE-seq [27] }\end{array}$ \\
\hline 3D chromatin structure & - & $\begin{array}{l}3 \mathrm{C}[28] \\
4 \mathrm{C}[29] \\
5 \mathrm{C}[30] \\
\mathrm{Hi}-\mathrm{C}[31] \\
\text { ChIA-PET [32] }\end{array}$ \\
\hline Non-coding RNA localization & $\begin{array}{l}\text { IncRNA } \\
\text { smRNA (siRNA, piRNA, miRNA) }\end{array}$ & $\begin{array}{l}\text { Deep sequencing of transcriptomes by mRNA-seq [33] } \\
\text { smRNA-seq [34] }\end{array}$ \\
\hline
\end{tabular}

MRE-seq, methylation-sensitive restriction enzyme sequencing; MeDIP-seq, methylated DNA immunoprecipitation sequencing; MBD-seq, methyl-CpG-binding domain protein sequencing; RRBS, reduced representation bisulfite sequencing; WGBS, whole-genome bisulfite sequencing; oxBS-seq, oxidative bisulfite sequencing; ChIP-seq, chromatin immunoprecipitation sequencing; ChIP-exo, chromatin immunoprecipitation-exonuclease; DGF, digital genomic footprint; FAIRE-seq, formaldehyde-assisted isolation of regulatory elements sequencing; ChIA-PET, chromatin interaction analysis by paired-end tag sequencing.

of this epigenetic mark revealed that in some instances DNA methylation is correlated with transcriptional activation, it is enriched in the gene bodies of active genes [37]. Thus, the associations between an epigenomic mark and functional output may be location-specific, thereby complicating their functional interpretation.

The available assays for identifying methylated $\mathrm{CpG}$ dinucleotides in a genome vary in terms of resolution and cost. Restriction enzyme-based assays (e.g., methylationsensitive restriction enzyme sequencing) involve the digestion of genomic DNA with several methylation-sensitive enzymes [15], followed by sequencing of digested fragments. Affinity-based enrichment assays, such as methylated DNA immunoprecipitation sequencing and methylCpG-binding domain protein sequencing $[16,17]$, selectively target methylated fragments (usually up to $100 \mathrm{bp}$ ) using an antibody, followed by sequencing of those fragments. Both these methods are low-resolution and essentially qualitative. Chemical modification methods, such as bisulfite sequencing (BS), have higher resolution and pro- vide quantitative estimates of methylation. They exploit the fact that when treated with sodium bisulfite, unmethylated cytosines convert to uracil while methylated cytosines do not. Thus, mapping of sequenced fragments reveals the quantitative estimate of methylation at each CpG locus. The two variations to $B S$ include reduced representation $B S$ and whole-genome bisulfite sequencing, or methylC-seq (wholegenome BS); the former employs restriction enzyme digestion to target certain regions of interest prior to sodium bisulfite treatment [18], whereas the latter couples the chemical treatment with whole-genome sequencing [19].

Upon the discovery that 5-hydroxymethylcytosine (5hmC: an intermediate produced during active DNA demethylation) and 5-methylcytosine (5-mC) are both resistant to bisulfite treatment, it became critical to distinguish between the different methyl variants, including 5-formylcytosine and 5-carboxylcytosine. To this end, oxidative bisulfite sequencing (oxBS-seq) was invented as an improvement for methylC-seq to measure single base-pair resolution methylation levels of 5-mC [20]. This is accomplished by 
oxidizing 5-hmCs (using potassium perruthenate) before sodium bisulfite treatment, such that they are now sensitive to the subjected chemical conversion to uracil, thus ensuring that the remaining cytosines are all 5-mCs.

\section{Histone modifications}

Chromosomal DNA is wrapped around histone octamers, essentially composed of 4 kinds of subunits; viz., H2A, H2B, $\mathrm{H} 3$, and H4. These proteins are subject to chemical modifications at specific residues of histone tails; some wellstudied modifications include phosphorylation, methylation, and ubiquitination [38]. These modifications are involved in setting the stage for directed transcriptional activation and repression by controlling DNA accessibility or recruitment of other protein complexes. This idea has been extended into the "histone code" hypothesis [39, 40] - that complex combinations of distinct histone modifications, like H3K27me3 (a mark of repressed regions), H3K4me3 (a mark of gene promoters), and H3K27ac (a mark of transcriptionally active regions), etc. [41], underlie specific transcriptional programs. This notion has been further extended in more recent works into an 'epigenomic code' to include epigenomic marks other than histone modifications [42].

Chromatin immunoprecipitation (ChIP) of DNA-histone complexes is achieved by cross-linking histones with the DNA, digesting the cross-linked DNA, and then using an antibody specific to the $\mathrm{N}$-tail modification of interest (to any of the $>100$ post-translational modifications to histone tails and globules [43]), followed by sequencing of the purified DNA fragments. This method is called chromatin immunoprecipitation sequencing (ChIP-seq) and was primarily used to map the genomic locations of DNA-associated proteins. Due to the relatively large fragment size, the mapped sequence reads thus obtained from ChIP-seq [21] provide a low-resolution localization map of modified histones in the genome. An alternative strategy, chromatin immunoprecipitation-exonuclease (ChIP-exo), uses exonuclease to digest the precipitated DNA fragment from either end to better resolve the modified loci at base-pair resolution [22].

\section{Nucleosome positioning and occupancy}

The packaging of DNA coiled around histones produces distinct structures called nucleosomes that form repeating units with approximately $147 \mathrm{bp}$ wrapped around each unit, separated by varying lengths of linker DNA. The occupancy and periodic positioning of nucleosomes can control the accessibility of DNA to transcription factors [23] and DNases, as well as the transcription rate of active gene bodies [44], and are thus considered an epigenetic mark.
Biochemically active regulatory regions are generally depleted of nucleosomes [45], whereas inactive repeat regions (heterochromatin) have higher affinity to form nucleosome structures [46].

A map of nucleosome occupancy is generated by using micrococcal nuclease (MNase) for digestion of the chromatin [23], followed by high-throughput sequencing-a technique called MNase-seq. MNase digests all linker DNA but preserves the DNA wound around the nucleosome; when the latter is sequenced and mapped to the reference, a map of the original positioning of nucleosomes is obtained. Due to some inherent biases in MNase affinity (preference to AT-rich regions) and the lack of single base-pair resolution data, attempts were made to develop more precise technologies [24]. An MNase-independent technique that involves chemically modifying engineered histones to bring about cleavage of DNA wound around histones allows direct mapping of nucleosome centers.

\section{Chromatin accessibility}

Epigenetic mechanisms, such as chromatin accessibility, impact transcription factor binding to DNA, transcriptional specificity, and hence, transcriptional regulation. Open and easily accessible regions of DNA within the chromatin are indicative of local territories of transcriptional activity. Measuring "openness" of DNA at different regions genomewide, based on the DNase hypersensitivity assay, has helped discover several classes of functional elements, like promoters and enhancers [47]. It has also aided in identifying cell-type specific behaviors by comparison of accessibility profiles [47].

Regions of accessible chromatin are reflective of active regulatory sites. The enzyme DNase I is capable of digesting DNA in nucleosome-depleted regions (i.e., free unwound DNA). Post-digestion sequencing reveals large blocks of DNase hypersensitive sites (DHS) in chromatin (DNaseseq) $[25,48]$, which, upon further deep sequencing, can reveal up to 40-bp footprints of protected regions (potentially bound by transcription factors). These smaller regions are called digital genomic footprints (DGF) [26]. Formaldehyde-assisted isolation of regulatory elements sequencing is another technique that, by deep-sequencing random fragments of the genome post-crosslinking, measures the frequency of shearing at different loci, thus quantifying accessibility at high resolution, because free DNA that is unbound by factors is more susceptible to shearing [27].

\section{D chromatin architecture}

The array of nucleosomes organized in 30-nm fibers, called chromatin, has diverse functions well beyond mere compaction. Coordinated activity of distal elements is 
orchestrated by short- and long-range DNA interactions, which is determined by the 3D chromatin structure as well as the local environment of individual genes. For instance, chromatin conformation/looping mediates a promoter's access to its enhancers, thereby determining the transcriptional fate of a gene [49]. Spatial proximity can be determined by measuring the interaction frequencies of linearly distal fragments by a suite of chromosome conformation capture (3C)-based assays.

Chromosomes assume a specific tertiary structure with profound implications for cellular function and fate [49]. While the overall chromatin structure cannot be directly measured, measuring spatial distances between pairs of genomic loci has become increasingly more efficient with the arrival of 3C technologies [28]. Treatment with formaldehyde crosslinks spatially proximal DNA loci in cells, and then post-digestion, the resulting fragments are allowed to ligate in a weak solution (cross-linked fragments ligate with higher frequency than random fragments). This frequency is quantitatively assessed by quantitative polymerase chain reaction. Known loci are selected for by using sequencespecific probes that allow assaying specific pairs of regions in so-called one-versus-one mapping. Circular chromosome conformation capture, or 4C, allows whole-genome mapping of all spatial interactions of one specific region of interest (one-vs-all mapping) [29]. Chromosome conformation capture carbon copy, or 5C, deploys several anchors and primers to ensure targeting of several regions at once to attain higher coverage (many-vs-many) [30]. Finally, the most recent derivative of $3 \mathrm{C}$, namely $\mathrm{Hi}-\mathrm{C}$, permits surveying of the whole genome [31] in a relatively unbiased fashion for frequencies of spatially interacting pairs of loci in the entire genome without restricting the exploration to selected loci. This was done with reasonably high resolution, where the human genome was partitioned into 100-kb blocks and the spatial interaction between every pair of intra-chromosomal regions was assessed, while interchromosomal interactions have been mapped at $1-\mathrm{Mb}$ resolution [31].

A variation of Hi-C, called chromatin interaction analysis by paired-end tag sequencing (ChIA-PET), involves a ChIP reaction to isolate chromatin interactions involving regions of DNA that bind to a protein of interest [32]. For example, an antibody targeting RNA polymerase II can be used to precipitate interactions involving a promoter and another interacting region, such as a distal enhancer or other promoters [50].

\section{Non-coding RNA localization}

Specific classes of non-coding RNA-short RNAs (viz. micro RNA [miRNA], short interfering RNA [siRNA], and piwi-interacting RNA [piRNA]) and long non-coding RNAs (lncRNA)-regulate gene expression through epigenetic mechanisms, influencing several cellular processes, like $\mathrm{X}$ chromosome inactivation [51], genomic imprinting [52], and some cellular processes [53], and cancer [54]. LncRNAs tether epigenetic complexes capable of methylating DNA and modifying histones to the chromatin, enabling alleleand locus-specific regulation. For example, lncRNA Xist covers the inactive $\mathrm{X}$ chromosome and recruits PCR2, a Polycomb complex responsible for trimethylation of $\mathrm{H} 3 \mathrm{~K} 27$ [51]. Furthermore, lncRNAs, by virtue of their length, are suited for orchestrating specific regulatory events particular to a target locus [55].

Additionally, miRNA ( $\sim 22$ nucleotides long) have shown to be linked with cancer and can act as either oncogenes or tumor-suppressor genes. They can be involved in establishing DNA methylation and regulating histone modification by interacting with their target mRNA to alter chromatin of the corresponding DNA template [56]. siRNA, and its interference machinery has also been implicated in the formation of heterochromatin [57].

A catalog of small RNAs has been generated by deep sequencing of total RNA from whole transcriptomes using smRNA-seq technology [34]. Selection of smRNA can be carried out by sequential ligation of adapters to its unique 5 mono/triphosphate ends, produced as a result of processing the smRNA population in the cell [58]. On the other hand, lncRNA are poly-adenylated, and much of the challenge in identifying them from whole-genome mRNA-seq data [33] lies in the computational and analytical domains [59].

\section{Epigenome Projects and Consortia}

Spurred by unprecedented advances in sequencing technologies as well as other assays, and a recognition of the potential of NGS in determining the epigenomic landscape, several research consortia have formed to generate genomewide maps of human epigenomic marks by sharing resources and publishing standard operating procedures, thereby ensuring best practices and high-quality datasets [60]. One of the first concerted efforts in this direction was the Encyclopedia of DNA Elements (ENCODE) Project, started in 2003 and funded by the National Human Genome Research Institute at the National Institute of Health $(\mathrm{NIH}-$ NHGRI), which involved hundreds of researchers in dozens of labs across the globe. The ENCODE consortium has performed 1,650 epigenomic profiling on 147 cell lines assessing the transcriptome, transcription factor binding for dozens of transcription factors, chromatin topology, histone modifications, DNA methylation, and more (http://www. encodeproject.org) [13]. Five years after launching 
ENCODE, the NIH funded a second large-scale mapping project called the Roadmap Epigenomics Program. While ENCODE focused on generating data in cell lines, the Roadmap program focuses on mapping epigenomes of high-priority normal human primary tissue and human embryonic stem cell (ESC)-derived cell types. As of May 2012, the Roadmap includes 61 "complete" epigenomes (of a variety of cell types) (http://www.roadmapepigenomics. org) [61]. The data repository can be accessed interactively at Human Epigenome Atlas (http://www.genboree.org/ epigenomeatlas/multiGridViewerPublic.rhtml), via the Washington University Epigenome Browser (http://ww w.epigenomegateway.wustl.edu/browser), as well as the UCSC Genome Browser (http://www.genome.ucsc.edu). Further, the NIH Roadmap is one of the members of an even larger consortium called the International Human Epigenome Consortium (IHEC; http://www.ihec-epigenomes. org), formed by institutions in 7 countries. To name a few, BLUEPRINT: a European initiative to generate about 100 reference epigenomic maps; the CREST/IHEC team in Japan: to produce reference epigenomes specializing in gastrointestinal epithelial cells, vascular endothelial cells, and cells of reproductive organs; and the Epigenomic Platform Program: a Canadian program to map epigenomic variations in disease cells. Together, these efforts are expected to generate over 1,000 reference human epigenomes. Other epigenome mapping efforts focused on specific disease areas have also been undertaken. These include the Cancer Genome Atlas (TCGA), funded by the NIH in 2006 [62], which is a part of the International Cancer Genome Consortium (ICGC; http://www.icgc.org) that was officially put together in 2008.

\section{What Have We Learned?}

By providing a rich functional annotation of the genome, the numerous epigenomic datasets have significantly improved our ability to probe the mechanisms of gene regulation as well as shed light on disease processes mediated by perturbation of normal regulatory processes. A collection of 30 scientific reports published with the initial release of the ENCODE data provides a detailed report of the initial integrative analysis of the epigenomics data [47, 63-66].

At a basic level, the data have revealed epigenomic signatures of a variety of functional elements, thus significantly enriching their annotation. For instance, many non-coding RNAs have been successfully annotated as a result of deciphering an epigenomic code specific to their promoters and gene bodies $[67,68]$. High-throughput profiling of the histone modification HeK4mel has revealed that it is a characteristic mark of enhancer elements; this, and other correlative marks have enabled the discovery of tens of thousands of cell-specific enhancers $[69,70]$ in the human genome, drastically improving our ability to probe the mechanisms of transcriptional regulation. In fact, chromatin signatures specific to promoters, enhancers, and repressed regions, etc., have all been modeled by computational methods like ChromHMM [42]. Furthermore, by isolating footprints of protected DNA present inside blocks of hypersensitive or nucleosome-depleted DNA, a comprehensive list of potential cis-regulatory binding sites/sequences has been identified, despite the lack of specific antibodies or the knowledge of cell-specific regulators [2].

Availability of epigenomic data has revived predictive modeling of condition-specific gene expression. For example, Dong et al. [71] generated a 2-step quantitative model that includes genome localization data for 11 histone modifications and 1 histone variant in 7 human cell lines and gene expression data quantified using RNA-seq and cap analysis gene expression (CAGE) in the nuclear and cytosolic compartments of the cell. The Pearson's correlation coefficient between their predicted and measured expression levels in about 78 experiments ranged between 0.6 and 0.9 (median $r=0.83$ ). Natarajan et al. [72] trained a classifier that associates cell-specific TF sequence motif matches in DHS regions with different expression patterns in 19 diverse cell types, thus predicting cell-type specific expression directly from regulatory elements in open chromatin.

Prior to the availability of NGS, details of the spatial structure of chromatin and its correlates, as well as its functional impacts were largely uncharted. Characterization of the spatial proximity of distal genomic elements with 3C-derivative assays has revealed a high rate of widespread promiscuous interactions among enhancers and promoters. For instance, more than $40 \%$ of promoters interact with multiple distal sites, and this is likely true for enhancers as well [73]. The spatial chromatin interaction data have also helped challenge some previously held beliefs that were nevertheless unsupported. For instance, historically, the DNA-binding protein CTCF was known to function, among other things, as an insulator that inhibited the spatial interaction between an enhancer and its target promoter when flanking a CTCF-bound locus. However, recent $5 \mathrm{C}$ data showed that almost $60 \%$ of all enhancer-promoter interactions happen, despite an intervening CTCF-bound locus [74]. In fact, there is increasing evidence that CTCF, along with cohesin is involved in coordinating long-range interactions between regulatory elements, and that these events are reflective of cell-type specific transcriptional programs [75]. 
With the availability of putative genome-wide enhancers along with their cell type-specific activity profile across numerous cell types, it is now possible to investigate an alternate layer of transcriptional regulation represented by a network of distal enhancers that exhibit correlated activities across cell types [76, 77] and jointly underlie correlated expression of functionally linked genes. Such analysis brings forth an alternative view of transcriptional regulation, where instead of a single gene regulated by one or more regulatory elements, one ought to consider the collective of enhancers and genes, co-localized in nuclear space to achieve coexpression of functionally linked genes [78, 79].

Epigenomic profiling along the developmental time course from ESCs to differentiated cells has shed light on transcriptional regulatory mechanisms during early development. For instance, comparison of the "histone code" between the two developmental timeframes indicates that regions marked by repressive histone marks are generally larger in differentiated cells and that these regions span developmental genes. It was also noted that the same regions are more accessible in ESCs [80, 81], thus suggesting an epigenome-mediated regulation of developmental gene transcription. Precisely how this is accomplished is yet to be clarified.

The epigenomic data and the discovery of significant patterns of epigenomic marks associated with specific functional regions have also helped clarify genotypephenotype association data. Numerous GWAS have revealed several polymorphic loci associated with various diseases [82]. However, a mechanistic interpretation of these signals has been hampered by the fact that most of the diseaseassociated genetic variants reside in non-coding regions with no obvious functional interpretation. By combining epigenomic maps and genetic variants, it was found that these disease-associated variants are enriched within cell typespecific accessible (DHS regions) and active regulatory regions [2]. Also, an epigenomic model of cardiac enhancers was shown to provide a better causal interpretation of GWAS signals associated with heart-related traits [83].

\section{Open Questions and Challenges}

The slew of epigenetic data has revolutionized our perception of the human genome. Although not all "epigenetically marked" regions of the genome are likely to be functional, it is very likely that a large portion is functionalmuch more than the $5-10 \%$ of the genome that is deemed to evolve under purifying selection [84, 85]. Analysis of transcriptional and epigenetic data has revealed that almost half of the genome is involved in carrying out specific biochemical functions [13]. Where these biochemical func- tions fit in the big picture, and how they are regulated are still open questions.

Another fundamental problem is that the causal relationships among various epigenomic events, such as DNA methylation, histone modifications, TF binding, and gene expression, etc., are currently not well understood. Although correlations between these events have been well documented in different contexts, we do not know if one is necessary and/or sufficient to observe another.

Before the full potential of NGS in mapping the epigenome can be attained, several technical challenges need to be overcome. For instance, techniques like ChIP-seq, used for profiling transcription factor binding and histone modifications require a large amount of starting material, ranging up to 5 million cells. Collection of such a large population of primary differentiated cells, progenitors, and those from specific developmental stages is inherently hard. Heterogeneity of a cell population is another issue that needs to be resolved. It is challenging to obtain a homogeneous population of cells of a singular type from primary tissues that often contain a heterogeneous population of cell types, and it is this lack of homogeneity that can adversely impact the interpretability and generalizability of the results obtained from analyzing the epigenomic data.

Yet, many solutions to tackle the problems above are underway. First, there is the emergence of techniques - like ChIP-nano (nano-scale ChIP), which is a highly sensitive small-scale ChIP-seq protocol with a requirement of $<50,000$ cells as starting material [86], and single-molecule real-time sequencing [87], which aims at alleviating cell-population effects during mapping specific features. Second, methods that are aimed at the targeted knock-out of epigenetic modifiers, like DNA methyltransferases and histone modification enzymes, at predetermined DNA sequences (using transcription activator-like effector nucleases [TALENs]) [88], can help to better characterize the functional relationships between gene expression and epigenetics, thus providing proof of concept for expression modulation (also called epigenetic editing methods) [89]. Also, powerful statistical approaches are being developed to integrate data $[42,90]$ to resolve some of these fundamental issues. With more epigenomic maps being profiled, and more effective computational approaches being developed, we are inching towards a holistic mechanistic understanding of cellular and organismal processes.

\section{Acknowledgments}

The authors would like to thank Justin Malin and Leonid Sukharnikov for their useful comments. This work was funded by the National Institutes of Health R01GM100335. 


\section{References}

1. U.S. Department of Health and Human Services. NIH News National Institues of Health. International Consortium Announces the 1000 Genomes Project. Bethesda: National Human Genome Research Institutes, 2008. Accessed 2014 Jan 1. Available from: http://www.nih.gov/news/health/ jan2008/nhgri-22.htm.

2. Maurano MT, Humbert R, Rynes E, Thurman RE, Haugen E, Wang $\mathrm{H}$, et al. Systematic localization of common disease-associated variation in regulatory DNA. Science 2012; 337:1190-1195.

3. King MC, Wilson AC. Evolution at two levels in humans and chimpanzees. Science 1975;188:107-116.

4. Inbar-Feigenberg M, Choufani S, Butcher DT, Roifman M, Weksberg R. Basic concepts of epigenetics. Fertil Steril 2013; 99:607-615.

5. Baker M. Genomics: Genomes in three dimensions. Nature 2011;470:289-294.

6. Ptashne M. Epigenetics: core misconcept. Proc Natl Acad Sci U S A 2013;110:7101-7103.

7. Bernstein BE, Meissner A, Lander ES. The mammalian epigenome. Cell 2007;128:669-681.

8. Berger SL, Kouzarides T, Shiekhattar R, Shilatifard A. An operational definition of epigenetics. Genes Dev 2009;23:781783.

9. Telese F, Gamliel A, Skowronska-Krawczyk D, Garcia-Bassets I, Rosenfeld MG. "Seq-ing" insights into the epigenetics of neuronal gene regulation. Neuron 2013;77:606-623.

10. Jaenisch R, Bird A. Epigenetic regulation of gene expression: how the genome integrates intrinsic and environmental signals. Nat Genet 2003;33 Suppl:245-254.

11. Bird A. DNA methylation patterns and epigenetic memory. Genes Dev 2002;16:6-21.

12. Koboldt DC, Steinberg KM, Larson DE, Wilson RK, Mardis ER. The next-generation sequencing revolution and its impact on genomics. Cell 2013;155:27-38.

13. ENCODE Project Consortium, Bernstein BE, Birney E, Dunham I, Green ED, Gunter C, et al. An integrated encyclopedia of DNA elements in the human genome. Nature 2012;489:57-74.

14. Turner BM. Defining an epigenetic code. Nat Cell Biol 2007; 9:2-6.

15. Laird PW. Principles and challenges of genomewide DNA methylation analysis. Nat Rev Genet 2010;11:191-203.

16. Down TA, Rakyan VK, Turner DJ, Flicek P, Li H, Kulesha E, et al. A Bayesian deconvolution strategy for immunoprecipitation-based DNA methylome analysis. Nat Biotechnol 2008; 26:779-785.

17. Serre D, Lee BH, Ting AH. MBD-isolated Genome Sequencing provides a high-throughput and comprehensive survey of DNA methylation in the human genome. Nucleic Acids Res 2010;38:391-399.

18. Meissner A, Gnirke A, Bell GW, Ramsahoye B, Lander ES, Jaenisch R. Reduced representation bisulfite sequencing for comparative high-resolution DNA methylation analysis. Nucleic Acids Res 2005;33:5868-5877.
19. Lister R, O'Malley RC, Tonti-Filippini J, Gregory BD, Berry $\mathrm{CC}$, Millar AH, et al. Highly integrated single-base resolution maps of the epigenome in Arabidopsis. Cell 2008;133: 523-536.

20. Booth MJ, Branco MR, Ficz G, Oxley D, Krueger F, Reik W, et al. Quantitative sequencing of 5-methylcytosine and 5-hydroxymethylcytosine at single-base resolution. Science 2012; 336:934-937.

21. Johnson DS, Mortazavi A, Myers RM, Wold B. Genome-wide mapping of in vivo protein-DNA interactions. Science 2007 316:1497-1502.

22. Rhee HS, Pugh BF. Comprehensive genome-wide protein-DNA interactions detected at single-nucleotide resolution. Cell 2011;147:1408-1419.

23. Bell O, Tiwari VK, Thomä NH, Schübeler D. Determinants and dynamics of genome accessibility. Nat Rev Genet 2011; 12:554-564.

24. Brogaard K, Xi L, Wang JP, Widom J. A map of nucleosome positions in yeast at base-pair resolution. Nature 2012;486: 496-501.

25. Crawford GE, Holt IE, Whittle J, Webb BD, Tai D, Davis S, et al. Genome-wide mapping of DNase hypersensitive sites using massively parallel signature sequencing (MPSS). Genome Res 2006;16:123-131.

26. Neph S, Vierstra J, Stergachis AB, Reynolds AP, Haugen E, Vernot $\mathrm{B}$, et al. An expansive human regulatory lexicon encoded in transcription factor footprints. Nature 2012;489: 83-90.

27. Giresi PG, Kim J, McDaniell RM, Iyer VR, Lieb JD. FAIRE (Formaldehyde-Assisted Isolation of Regulatory Elements) isolates active regulatory elements from human chromatin. Genome Res 2007;17:877-885.

28. Dekker J, Rippe K, Dekker M, Kleckner N. Capturing chromosome conformation. Science 2002;295:1306-1311.

29. Zhao Z, Tavoosidana G, Sjölinder M, Göndör A, Mariano P, Wang $S$, et al. Circular chromosome conformation capture (4C) uncovers extensive networks of epigenetically regulated intra- and interchromosomal interactions. Nat Genet 2006 38:1341-1347.

30. Dostie J, Richmond TA, Arnaout RA, Selzer RR, Lee WL, Honan TA, et al. Chromosome Conformation Capture Carbon Copy (5C): a massively parallel solution for mapping interactions between genomic elements. Genome Res 2006;16: 1299-1309.

31. Lieberman-Aiden E, van Berkum NL, Williams L, Imakaev M, Ragoczy T, Telling A, et al. Comprehensive mapping of long-range interactions reveals folding principles of the human genome. Science 2009;326:289-293.

32. Fullwood MJ, Liu MH, Pan YF, Liu J, Xu H, Mohamed YB, et al. An oestrogen-receptor-alpha-bound human chromatin interactome. Nature 2009;462:58-64.

33. Derrien T, Johnson R, Bussotti G, Tanzer A, Djebali S, Tilgner $\mathrm{H}$, et al. The GENCODE $\mathrm{v}$ catalog of human long noncoding RNAs: analysis of their gene structure, evolution, and expression. Genome Res 2012;22:1775-1789.

34. Zhou L, Li X, Liu Q, Zhao F, Wu J. Small RNA transcriptome investigation based on next-generation sequencing techno- 
logy. J Genet Genomics 2011;38:505-513

35. Tucker KL. Methylated cytosine and the brain: a new base for neuroscience. Neuron 2001;30:649-652.

36. Newell-Price J, Clark AJ, King P. DNA methylation and silencing of gene expression. Trends Endocrinol Metab 2000;11: 142-148.

37. Lister R, Pelizzola M, Dowen RH, Hawkins RD, Hon G, Tonti-Filippini J, et al. Human DNA methylomes at base resolution show widespread epigenomic differences. Nature 2009; 462:315-322.

38. Bártová E, Krejcí J, Harnicarová A, Galiová G, Kozubek S. Histone modifications and nuclear architecture: a review. $J$ Histochem Cytochem 2008;56:711-721.

39. Strahl BD, Allis CD. The language of covalent histone modifications. Nature 2000;403:41-45.

40. Jenuwein T, Allis CD. Translating the histone code. Science 2001;293:1074-1080.

41. Bannister AJ, Kouzarides T. Regulation of chromatin by histone modifications. Cell Res 2011;21:381-395.

42. Ernst J, Kellis M. ChromHMM: automating chromatin-state discovery and characterization. Nat Methods 2012;9:215-216.

43. Tian Z, Tolić N, Zhao R, Moore RJ, Hengel SM, Robinson EW, et al. Enhanced top-down characterization of histone post-translational modifications. Genome Biol 2012;13:R86.

44. Bintu L, Ishibashi T, Dangkulwanich M, Wu YY, Lubkowska L, Kashlev M, et al. Nucleosomal elements that control the topography of the barrier to transcription. Cell 2012;151:738749.

45. Lee CK, Shibata Y, Rao B, Strahl BD, Lieb JD. Evidence for nucleosome depletion at active regulatory regions genome-wide. Nat Genet 2004;36:900-905.

46. Trifonov EN. Cracking the chromatin code: precise rule of nucleosome positioning. Phys Life Rev 2011;8:39-50.

47. Thurman RE, Rynes E, Humbert R, Vierstra J, Maurano MT, Haugen $\mathrm{E}$, et al. The accessible chromatin landscape of the human genome. Nature 2012;489:75-82.

48. Boyle AP, Davis S, Shulha HP, Meltzer P, Margulies EH, Weng $Z$, et al. High-resolution mapping and characterization of open chromatin across the genome. Cell 2008;132:311-322.

49. Harmston N, Lenhard B. Chromatin and epigenetic features of long-range gene regulation. Nucleic Acids Res 2013;41:71857199.

50. de Wit E, de Laat W. A decade of 3C technologies: insights into nuclear organization. Genes Dev 2012;26:11-24.

51. Zhao J, Sun BK, Erwin JA, Song JJ, Lee JT. Polycomb proteins targeted by a short repeat RNA to the mouse X chromosome. Science 2008;322:750-756.

52. Koerner MV, Pauler FM, Huang R, Barlow DP. The function of non-coding RNAs in genomic imprinting. Development 2009; 136:1771-1783.

53. Loewer S, Cabili MN, Guttman M, Loh YH, Thomas K, Park $\mathrm{IH}$, et al. Large intergenic non-coding RNA-RoR modulates reprogramming of human induced pluripotent stem cells. Nat Genet 2010;42:1113-1117.

54. Tsai MC, Spitale RC, Chang HY. Long intergenic noncoding RNAs: new links in cancer progression. Cancer Res 2011; $71: 3-7$
55. Lee JT. Epigenetic regulation by long noncoding RNAs. Science 2012;338:1435-1439.

56. Bao N, Lye KW, Barton MK. MicroRNA binding sites in Arabidopsis class III HD-ZIP mRNAs are required for methylation of the template chromosome. Dev Cell 2004;7:653-662.

57. Fukagawa T, Nogami M, Yoshikawa M, Ikeno M, Okazaki T, Takami Y, et al. Dicer is essential for formation of the heterochromatin structure in vertebrate cells. Nat Cell Biol 2004; 6:784-791.

58. Havecker ER. Detection of small RNAs and microRNAs using deep sequencing technology. Methods Mol Biol 2011;732: 55-68.

59. Ilott NE, Ponting CP. Predicting long non-coding RNAs using RNA sequencing. Methods 2013;63:50-59.

60. Landt SG, Marinov GK, Kundaje A, Kheradpour P, Pauli F, Batzoglou S, et al. ChIP-seq guidelines and practices of the ENCODE and modENCODE consortia. Genome Res 2012; 22:1813-1831.

61. Bernstein BE, Stamatoyannopoulos JA, Costello JF, Ren B, Milosavljevic A, Meissner A, et al. The NIH Roadmap Epigenomics Mapping Consortium. Nat Biotechnol 2010;28: 1045-1048.

62. Garraway LA, Lander ES. Lessons from the cancer genome. Cell 2013;153:17-37.

63. Wang J, Zhuang J, Iyer S, Lin X, Whitfield TW, Greven MC, et al. Sequence features and chromatin structure around the genomic regions bound by 119 human transcription factors. Genome Res 2012;22:1798-1812.

64. Gerstein MB, Kundaje A, Hariharan M, Landt SG, Yan KK, Cheng $\mathrm{C}$, et al. Architecture of the human regulatory network derived from ENCODE data. Nature 2012;489:91-100.

65. Yip KY, Cheng C, Bhardwaj N, Brown JB, Leng J, Kundaje A, et al. Classification of human genomic regions based on experimentally determined binding sites of more than 100 transcription-related factors. Genome Biol 2012;13:R48.

66. Vernot B, Stergachis AB, Maurano MT, Vierstra J, Neph S, Thurman RE, et al. Personal and population genomics of human regulatory variation. Genome Res 2012;22:1689-1697.

67. Khalil AM, Guttman M, Huarte M, Garber M, Raj A, Rivea Morales $\mathrm{D}$, et al. Many human large intergenic noncoding RNAs associate with chromatin-modifying complexes and affect gene expression. Proc Natl Acad Sci U S A 2009;106: 11667-11672.

68. Guttman M, Amit I, Garber M, French C, Lin MF, Feldser D, et al. Chromatin signature reveals over a thousand highly conserved large non-coding RNAs in mammals. Nature 2009; 458:223-227.

69. Heintzman ND, Hon GC, Hawkins RD, Kheradpour P, Stark A, Harp LF, et al. Histone modifications at human enhancers reflect global cell-type-specific gene expression. Nature 2009; 459:108-112.

70. Rajagopal N, Xie W, Li Y, Wagner U, Wang W, Stamatoyannopoulos J, et al. RFECS: a random-forest based algorithm for enhancer identification from chromatin state. PLoS Comput Biol 2013;9:e1002968.

71. Dong X, Greven MC, Kundaje A, Djebali S, Brown JB, Cheng $\mathrm{C}$, et al. Modeling gene expression using chromatin features in 
various cellular contexts. Genome Biol 2012;13:R53.

72. Natarajan A, Yardimci GG, Sheffield NC, Crawford GE, Ohler U. Predicting cell-type-specific gene expression from regions of open chromatin. Genome Res 2012;22:1711-1722.

73. Sanyal A, Lajoie BR, Jain G, Dekker J. The long-range interaction landscape of gene promoters. Nature 2012;489:109113.

74. DeMare LE, Leng J, Cotney J, Reilly SK, Yin J, Sarro R, et al. The genomic landscape of cohesin-associated chromatin interactions. Genome Res 2013;23:1224-1234.

75. Merkenschlager M, Odom DT. CTCF and cohesin: linking gene regulatory elements with their targets. Cell 2013;152: 1285-1297.

76. Malin J, Aniba MR, Hannenhalli S. Enhancer networks revealed by correlated DNAse hypersensitivity states of enhancers. Nucleic Acids Res 2013;41:6828-6838.

77. Sheffield NC, Thurman RE, Song L, Safi A, Stamatoyannopoulos JA, Lenhard B, et al. Patterns of regulatory activity across diverse human cell types predict tissue identity, transcription factor binding, and long-range interactions. Genome Res 2013; 23:777-788.

78. Li G, Ruan X, Auerbach RK, Sandhu KS, Zheng M, Wang P, et al. Extensive promoter-centered chromatin interactions provide a topological basis for transcription regulation. Cell 2012; 148:84-98.

79. Sandhu KS, Li G, Poh HM, Quek YL, Sia YY, Peh SQ, et al. Large-scale functional organization of long-range chromatin interaction networks. Cell Rep 2012;2:1207-1219.

80. Zhu J, Adli M, Zou JY, Verstappen G, Coyne M, Zhang X, et al. Genome-wide chromatin state transitions associated with developmental and environmental cues. Cell 2013;152:642-654.

81. Stergachis AB, Neph S, Reynolds A, Humbert R, Miller B,
Paige SL, et al. Developmental fate and cellular maturity encoded in human regulatory DNA landscapes. Cell 2013;154: 888-903.

82. Hindorff LA, MacArthur J, Morales J, Junkins HA, Hall PN, Klemm AK, et al. A catalog of published genome-wide association studies. Bethesda: National Human Genome Research Institute, 2013. Accessed 2014 Jan 1. Available from: http://www.genome.gov/gwastudies.

83. Sahu AD, Aniba R, Chang YP, Hannenhalli S. Epigenomic model of cardiac enhancers with application to genome wide association studies. Pac Symp Biocomput 2013:92-102.

84. Ward LD, Kellis M. Evidence of abundant purifying selection in humans for recently acquired regulatory functions. Science 2012;337:1675-1678.

85. Lindblad-Toh K, Garber M, Zuk O, Lin MF, Parker BJ, Washietl $\mathrm{S}$, et al. A high-resolution map of human evolutionary constraint using 29 mammals. Nature 2011;478:476-482.

86. Adli M, Bernstein BE. Whole-genome chromatin profiling from limited numbers of cells using nano-ChIP-seq. Nat Protoc 2011;6:1656-1668.

87. Roberts RJ, Carneiro MO, Schatz MC. The advantages of SMRT sequencing. Genome Biol 2013;14:405.

88. Boch J. TALEs of genome targeting. Nat Biotechnol 2011; 29:135-136

89. de Groote ML, Verschure PJ, Rots MG. Epigenetic Editing: targeted rewriting of epigenetic marks to modulate expression of selected target genes. Nucleic Acids Res 2012;40:10596-10613.

90. Ke X, Cortina-Borja M, Silva BC, Lowe R, Rakyan V, Balding D. Integrated analysis of genome-wide genetic and epigenetic association data for identification of disease mechanisms. Epigenetics 2013;8:1236-1244. 